\title{
The permeability transition pore triggers Bax translocation to mitochondria during neuronal apoptosis
}

\author{
TA Precht ${ }^{1,2}$, RA Phelps ${ }^{1,2}$, DA Linseman ${ }^{1}$, BD Butts $^{1}$, SS Le $^{1}$, \\ TA Laessig ${ }^{1}$, RJ Bouchard ${ }^{1}$ and KA Heidenreich ${ }^{*, 1}$ \\ 1 Department of Pharmacology, University of Colorado Health Sciences Center \\ and the Denver Veterans Affairs Medical Center, Denver, CO, USA \\ 2 These authors contributed equally to this paper \\ * Corresponding author: KA Heidenreich, Department of Pharmacology, University \\ of Colorado Health Sciences Center at Fitzsimons, PO Box 6511, Mail stop \\ 8303, Aurora, CO 80045-0511, USA; \\ E-mail: Kim.Heidenreich@UCHSC.edu
}

Received 07.7.04; revised 26.10.04; accepted 02.11.04; published online 07.1.05 Edited by $Y$ Tsujimoto

\begin{abstract}
Cerebellar granule neurons (CGNs) require depolarization for their survival in culture. When deprived of this stimulus, CGNs die via an intrinsic apoptotic cascade involving Bim induction, Bax translocation, cytochrome $c$ release, and caspase- 9 and -3 activation. Opening of the mitochondrial permeability transition pore (mPTP) is an early event during intrinsic apoptosis; however, the precise role of $\mathrm{mPTP}$ opening in neuronal apoptosis is presently unclear. Here, we show that mPTP opening acts as an initiating event to stimulate Bax translocation to mitochondria. A C-terminal ( $\alpha 9$ helix) GFP-Bax point mutant (T182A) that constitutively localizes to mitochondria circumvents the requirement for MPTP opening and is entirely sufficient to induce CGN apoptosis. Collectively, these data indicate that the major role of MPTP opening in CGN apoptosis is to trigger $\mathrm{Bax}$ translocation to mitochondria, ultimately leading to cytochrome $c$ release and caspase activation.

Cell Death and Differentiation (2005) 12, 255-265.

doi:10.1038/sj.cdd.4401552

Published online 7 January 2005
\end{abstract}

Keywords: cerebellar granule neurons; cytochrome $c$; cyclosporin A; Bcl-2 family

Abbreviations: CGNs, cerebellar granule neurons; $\mathrm{BH}, \mathrm{Bcl}-2$ homology; mPTP, mitochondrial permeability transition pore; VDAC, voltage-dependent anion channel; ANT, adenine nucleotide translocator; CyA, cyclosporin A; TMRE, tetramethylrhodamine ethyl ester; COX IV, cytochrome $c$ oxidase subunit IV; HEK, human embryonic kidney; IGF-I, insulin-like growth factor-l; DAPI, 4',6-diamidino-2-phenylindole

\section{Introduction}

The normal development of the nervous system involves generation, as well as, degeneration of neurons to maintain tissue homeostasis. ${ }^{1}$ The balance of these processes is controlled in part by apoptosis, a type of programmed cell death. Dysregulated apoptosis is thought to contribute to a variety of neurodegenerative diseases. ${ }^{2}$ An established in vitro model for investigating the pathways of neuronal apoptosis is cultured rat cerebellar granule neurons (CGNs). ${ }^{3}$ When cultured CGNs are deprived of serum and depolarizing extracellular potassium, cell death proceeds by an intrinsic, mitochondrial cascade that involves cytochrome $c$ release and caspase activation. ${ }^{4,5}$

The intrinsic apoptotic pathway is regulated by the Bcl-2 family of proteins. ${ }^{6} \mathrm{Bcl}-2$ family members possess one or more of four Bcl-2 homology $(\mathrm{BH})$ domains. The precise complement of these structural domains determines the proor antiapoptotic activity of $\mathrm{Bcl}-2$-related proteins. ${ }^{7}$ For example, family members that possess exclusively $\mathrm{BH} 3$ domains are proapoptotic, and have multiple mechanisms of action. Some BH3-only proteins, such as Bad and Bik, promote the intrinsic death pathway by sequestering $\mathrm{Bcl}-2$ and inhibiting its antiapoptotic effects ${ }^{8}$ by displacing Bid and Bim. ${ }^{9}$ Bid and Bim can act directly to activate Bax, a multidomain proapoptotic $\mathrm{Bcl}-2$ family member. ${ }^{10}$ In response to undefined apoptotic signals, Bax translocates to the mitochondria where it oligomerizes into a heteromeric protein channel. This putative Bax channel inserts into the outer mitochondrial membrane and releases apoptotic factors, such as cytochrome $c$, eventually leading to caspase activation and cell death. ${ }^{11}$

The intrinsic apoptotic pathway is also regulated by the mitochondrial permeability transition pore (mPTP). ${ }^{12}$ The mPTP is thought to be a large protein complex composed of the voltage-dependent anion channel (VDAC), the adenine nucleotide translocator (ANT), and cyclophilin D. ${ }^{13} \mathrm{~A}$ recent study in hepatocytes has shown that the ANT may not be required to form a functional MPTP, but might play a role in regulation of the pore. ${ }^{14}$ The components of the MPTP are associated with the mitochondrial inner membrane and form a pore that is permeable to low molecular weight $(\leqslant 1500 \mathrm{Da})$ solutes and ions, ultimately leading to mitochondrial membrane depolarization and swelling of the mitochondrial matrix..$^{15}$ Whether $\mathrm{BH} 3$-only proteins are directly involved in the formation or function of the MPTP during neuronal apoptosis has not been elucidated. Likewise, the potential role of the MPTP in the regulation of Bax translocation during neuronal apoptosis is presently unclear.

De Giorgi et al. ${ }^{16}$ have previously shown that formation of the mPTP indirectly signals Bax to move to mitochondria in the non-neuronal COS cell line. Under these conditions, the release of cytochrome $c$ is dependent upon Bax movement, multimer formation, clustering, and insertion into the outer mitochondrial membrane. In contrast, in glioma cells, MPTP opening, indicated by a loss of mitochondrial membrane potential and matrix swelling, does not occur until very late 
phases of cell death after cytochrome $c$ release. ${ }^{17}$ Similarly, Krohn et al. ${ }^{18}$ previously showed that staurosporine-induced cytochrome $c$ release and caspase activation occurs in hippocampal neurons prior to any reduction in mitochondrial membrane potential. These studies indicate that the MPTP may or may not influence Bax-mediated cytochrome $c$ release in neurons.

In the current study, we investigated the role of the MPTP in localizing Bax to mitochondria in CGNs undergoing apoptosis. We utilized two specific tools to investigate the involvement of the mPTP in Bax translocation. Firstly, we used the MPTP inhibitor, cyclosporin $A(C y A)$, which binds cyclophilin $D$ and prevents pore formation. Secondly, we used a C-terminal point mutant of $\operatorname{Bax} \alpha(\mathrm{T} 182 \mathrm{~A})$ that constitutively localizes to mitochondria and induces apoptosis. We show that MPTP opening is an initiating event to stimulate Bax translocation to mitochondria. Moreover, constitutive localization of Bax to mitochondria circumvents the requirement for MPTP opening and is sufficient to induce apoptosis.

\section{Results}

\section{CyA blocks opening of the MPTP in CGNs subjected to trophic factor withdrawal}

Primary CGNs deprived of serum and depolarizing extracellular potassium (trophic factor withdrawal) die via activation of a mitochondrial apoptotic cascade. ${ }^{5}$ Profound mitochondrial swelling and loss of mitochondrial membrane potential are early events in CGN apoptosis that are consistent with opening of the MPTP, but the role of these mitochondrial changes in the execution of the apoptotic cascade is unclear. CyA inhibits opening of the MPTP by binding to cyclophilin D, an integral protein in the mPTP. ${ }^{19}$ Initially, we evaluated the utility of CyA as a pharmacological tool to prevent the mitochondrial swelling and depolarization induced by trophic factor withdrawal in CGNs. CGNs incubated in control medium (containing serum and depolarizing potassium; $25 \mathrm{~K}+$ Ser) displayed small, compact mitochondria visualized by staining with the cationic dye JC-1 (Figure 1a, left panel). In addition, CGNs maintained in control medium showed an intact mitochondrial membrane potential demonstrated by substantial tetramethylrhodamine ethyl ester (TMRE) staining (Figure 1b, left panel). When CGNs were switched for $4 \mathrm{~h}$ to apoptotic medium (lacking serum and depolarizing potassium; 5K-Ser), they displayed marked mitochondrial swelling (Figure 1a, middle panel) and a significant loss of mitochondrial membrane potential indicated by a substantial reduction in TMRE staining (Figure 1b, middle panel). The addition of CyA $(10 \mu \mathrm{M})$, at the time of trophic factor withdrawal, completely blocked both mitochondrial swelling (Figure 1a, right panel) and mitochondrial depolarization (Figure 1b, right panel), consistent with its known actions as an MPTP inhibitor. To further support the CyA effect on MPTP opening, we utilized a technique involving the cell-permeable dye calceinAM, which upon hydrolysis by cell esterases produces a green fluorescent dye. The calcein $/ \mathrm{Co}^{2+}$ assay for PTP opening was adapted from Petronilli et al. ${ }^{20}$ to visualize selective accumulation of calcein in mitochondria by quenching cytosolic and nuclear fluorescence with $\mathrm{Co}^{2+}$ addition. The
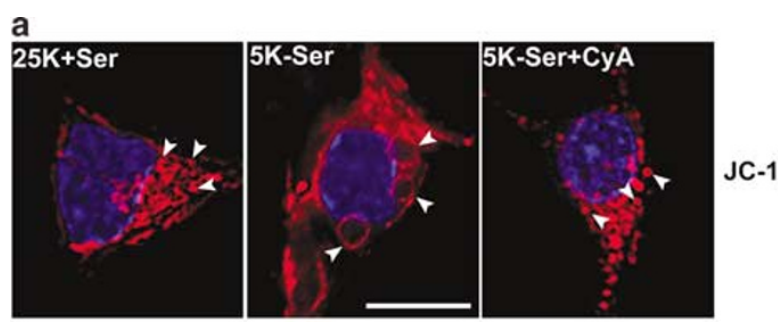

b
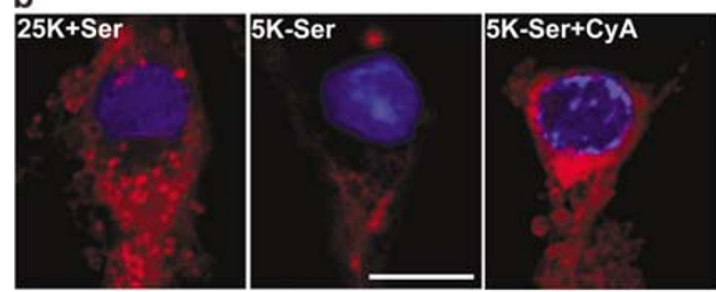

C
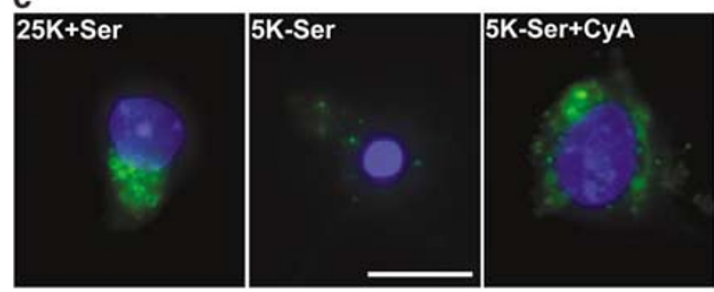

TMRE

Figure 1 Trophic factor withdrawal induces CyA-sensitive mitochondrial swelling and mitochondrial membrane depolarization. Mitochondrial swelling and depolarization are indicative of the opening of the PTP. Both the marked mitochondrial swelling and depolarization are blocked by the PTP inhibitor CyA. CGNs were incubated for $4 \mathrm{~h}$ in either control medium containing serum and depolarizing $(25 \mathrm{mM})$ extracellular potassium $(25 \mathrm{~K}+$ Ser) or apoptotic medium lacking serum and containing $5 \mathrm{mM}$ extracellular potassium (5K-Ser) in the absence or presence of CyA $(10 \mu \mathrm{M})$. (a) At 30 min prior to fixation, JC1 (final concentration, $2 \mu \mathrm{g} / \mathrm{ml}$ ) and Hoechst dye were added to the cultures to stain mitochondria and nuclei, respectively. JC1 fluorescence was captured using a Cy3 filter under $a \times 100$ oil objective. Mitochondria are indicated by the arrows. Note that CyA completely blocks the marked swelling of mitochondria induced by trophic factor deprivation. The images shown are representative of results obtained from four separate experiments. Scale bar, $10 \mu \mathrm{m}$. (b) CGNs were incubated exactly as described in (a). At $30 \mathrm{~min}$ before the end of the incubation period, tetramethylrhodamine ethylester (TMRE, $500 \mathrm{nM}$ ) and Hoechst were added directly to the cells. After incubation, the coverslips were inverted onto slides into a small volume of PBS containing glucose and $p$-phenylenediamine (antiquenching agent). Then, living cells were imaged under a $\times 100$ oil objective. Nuclear staining is shown in blue; TMRE is shown in red. CGNs maintained in either control medium or apoptotic medium containing CyA displayed many distinct mitochondria with intense TMRE staining, indicative of an intact mitochondrial membrane potential (left and right panels). In contrast, CGNs incubated in apoptotic medium alone (middle panel) showed only diffuse TMRE staining, indicative of mitochondrial depolarization. The images shown are representative of three separate experiments. Scale bar, $10 \mu \mathrm{m}$. (c) CGNs were incubated exactly as described in (a). At 20 min prior to the end of the incubation period, cells were loaded with $1 \mu \mathrm{M}$ calcein-AM in the presence of $1 \mathrm{mM} \mathrm{Co}^{2+}$, $\sim 0.1 \%$ Pluronic F-127, and Hoechst dye. After incubation, cells were washed and mounted on slides in a small volume of PBS containing glucose and $p$ phenylenediamine. Living cells were imaged under a $\times 100$ oil objective. Nuclear staining is shown in blue; calcein fluorescence is in green. The majority of CGNs maintained in control medium or apoptotic medium containing CyA displayed green fluorescence in distinct subcellular fractions, which was confirmed to be mitochondrial (see Materials and Methods) (left and right panels). However, CGNs maintained in apoptotic medium alone (middle panel) showed a dramatic loss of mitochondrial calcein fluorescence indicating an open mPTP. Images are representative of four separate experiments. Scale bar, $10 \mu \mathrm{m}$ 
left panel of Figure 1c illustrates that calcein fluorescence can be localized to mitochondria under control conditions $(25 \mathrm{~K}+$ Ser $)$, which was confirmed by colocalization with TMRE (data not shown). Upon trophic factor withdrawal (5K-Ser), calcein fluorescence is substantially decreased in cells undergoing apoptosis (Figure 1c, middle panel) and this decrease can then be reversed by addition of CyA (Figure 1c right panel). Multiple regions of interest were visualized to confirm the loss and rescue of calcein fluorescence of $5 \mathrm{~K}-\mathrm{Ser}$ and $5 \mathrm{~K}-\mathrm{Ser}+\mathrm{CyA}$, respectively (data not shown). These data indicate that trophic factor withdrawal induces PTP opening in mitochondria and that this opening can be blocked by CyA. In addition to binding cyclophilin D, CyA inhibits the type 2B Ser/Thr phosphatase, calcineurin, which in some cells dephosphorylates and activates the proapoptotic protein Bad, an action that is mimicked by FK506. ${ }^{21} \mathrm{FK} 506(10 \mu \mathrm{M})$ had no effect on either mitochondrial swelling or depolarization induced in CGNs incubated in apoptotic medium (data not shown), suggesting that the ability of CyA to block mPTP opening triggered by trophic factor withdrawal in CGNs is independent of its ability to inhibit calcineurin phosphatase activity.

\section{CyA inhibits cytochrome $c$ release in CGNs subjected to trophic factor withdrawal: the effects of CyA are independent of Bim regulation}

Cytochrome $c$ release is a key event in the activation of the intrinsic death pathway. ${ }^{13,22}$ Once released from mitochondria, cytochrome $c$ interacts with apaf- 1 and pro-caspase- 9 to form an apoptosome that ultimately activates the intrinsic initiator caspase- $9 .{ }^{23}$ Caspase- 9 subsequently cleaves the executioner caspase-3 resulting in the proteolysis of critical cellular proteins and apoptotic cell death. ${ }^{24}$ To determine if opening of the MPTP is required for cytochrome $c$ release in trophic factor-deprived CGNs, we analyzed the distribution of cytochrome $c$ in subcellular fractions. In CGNs maintained in control medium, cytochrome $c$ was localized predominantly in the mitochondrial fraction (Figure 2a, left two lanes). Following $6 \mathrm{~h}$ of trophic factor deprivation, a substantial portion of the cytochrome $c$ was released from mitochondria and redistributed into the cytosolic fraction (Figure $2 \mathrm{a}$, middle two lanes). Addition of CyA to CGNs incubated in apoptotic medium completely blocked the release of cyt $c$ from the mitochondria into the cytosol (Figure $2 a$, right two lanes). The enrichment of the cytosolic and mitochondrial fractions was verified by immunoblotting for the integral mitochondrial membrane protein, cytochrome $c$ oxidase subunit IV (COX IV) (Figure 2a, lower blot). These results indicate that inhibition of the MPTP with CyA prevents the release of cyt $c$ from mitochondria in trophic factor-deprived CGNs.

Recently, induction of the BH3-only protein, Bim, has been shown to be critical for intrinsic neuronal apoptosis. .,25,26 Immunoblot analysis of CGN extracts for Bim demonstrated that the expression of the short isoform of $\mathrm{Bim}\left(\mathrm{Bim}_{\mathrm{s}}\right.$, $\sim 15 \mathrm{kDa}$ ) was very low in control conditions and increased markedly upon induction of apoptosis (Figure $2 \mathrm{~b}$, first and second lanes). We previously reported that insulin-like growth factor-I (IGF-I), a prosurvival factor for CGNs in vitro and in

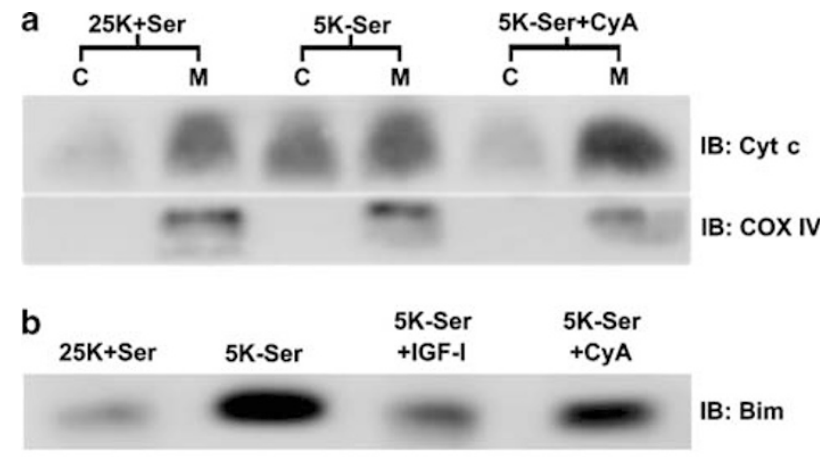

C

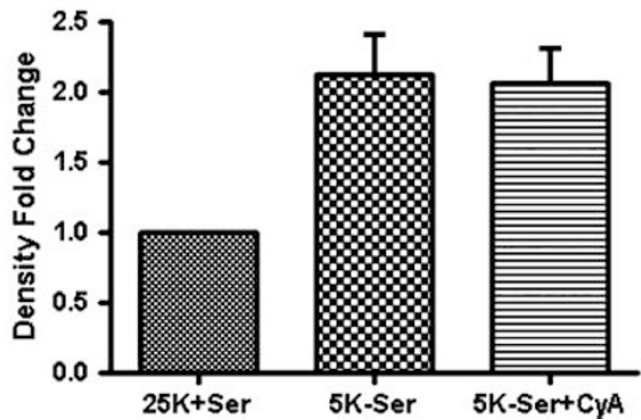

Figure 2 CyA inhibits cyt $c$ release in trophic factor-deprived CGNs independent of effects on Bim. (a) CGNs were incubated as described in Figure 1, but for $6 \mathrm{~h}$. After incubation, the cells were lysed and fractionated to separate the mitochondrial fraction (M) from the cytosolic fraction (C). The subcellular fractions were then subjected to SDS-PAGE on $15 \%$ polyacrylamide gels, and the proteins were transferred to PVDF membranes. Membranes were immunoblotted (IB) with an antibody to cytochrome $c$ (cyt $c$ ). The blots were then stripped and reprobed with an antibody to COX IV as a specific marker for the mitochondrial fractions. The blots shown are typical of results obtained in three separate experiments. (b) CGNs were incubated for $6 \mathrm{~h}$ in either control $(25 \mathrm{~K}+$ Ser) or apoptotic ( $5 \mathrm{~K}$-Ser) medium in the absence or presence of IGF-I $(200 \mathrm{ng} / \mathrm{ml})$ or CyA $(10 \mu \mathrm{M})$. After incubation, cells were lysed and CGN lysates were immunoblotted with an antibody that recognizes Bim. (c) Immunoblots of Bim were analyzed for densitometry and normalized to $25 \mathrm{~K}+$ Ser. The bar graph represents the fold change of density for $5 \mathrm{~K}-\mathrm{Ser}$ and $5 \mathrm{~K}-\mathrm{Ser}+\mathrm{CyA}(10 \mu \mathrm{M})$. There was no significant difference between $5 \mathrm{~K}-\mathrm{Ser}$ and $5 \mathrm{~K}-\mathrm{Ser}+\mathrm{CyA}$ as determined by ANOVA

vivo, ${ }^{27,28}$ inhibits CGN apoptosis without blocking either mitochondrial swelling or depolarization. ${ }^{5}$ A primary mechanism underlying the protective effects of IGF-I is its ability to block the increased expression of $\mathrm{Bim}_{\mathrm{s}}$ induced by trophic factor withdrawal in CGNs (Figure $2 b$, third lane). To exclude the possibility that CyA blocks cytochrome $c$ release by similarly influencing the expression of Bim, we examined CyA effects on Bim induction in CGNs incubated in apoptotic medium. Although both IGF-I ${ }^{5}$ and CyA (Figure 2a) inhibit cytochrome $c$ release, CyA did not substantially block the induction of $\mathrm{Bim}_{\mathrm{s}}$ in apoptotic medium (Figure 2b, fourth lane). Densitometric measurements of Bim immunoblots (Figure 2c) show an approximate two-fold increase in Bim expression in $5 \mathrm{~K}$-Ser medium when normalized to $25 \mathrm{~K}+$ Ser. There was no significant change in Bim expression when CyA was included in the medium. Collectively, these data suggest that there are MPTP-dependent (CyA-sensitive) and Bim-dependent (IGF-I-sensitive) mechanisms that act in concert to trigger cytochrome $c$ release in trophic factor-deprived CGNs. 


\section{Translocation of GFP-Bax to mitochondria during} CGN apoptosis is inhibited by CyA

Since CyA had no significant effect on the induction of Bim, we investigated another potential mechanism by which the MPTP could regulate cytochrome $c$ release in CGNs. We hypothesized that opening of the MPTP may trigger Bax translocation to mitochondria in trophic factor-deprived CGNs. To examine this question, we utilized a gene gun transfection method ${ }^{29}$ to transiently transfect primary CGNs with a construct encoding for a green fluorescent protein (GFP)-Bax fusion protein. ${ }^{30}$ We found that addition of CyA to GFP-Bax-transfected CGNs at the time of trophic factor withdrawal significantly blunted translocation of GFP-Bax to mitochondria (Figure 3a). Quantitative analysis showed that addition of CyA to apoptotic medium sustained a subcellular distribution of GFP-Bax that was indistinguishable from that observed in CGNs maintained in control medium (Figure $3 b$ ). In control conditions, $73 \pm 2 \%$ of GFP-Bax-expressing cells demonstrated a diffuse localiza-
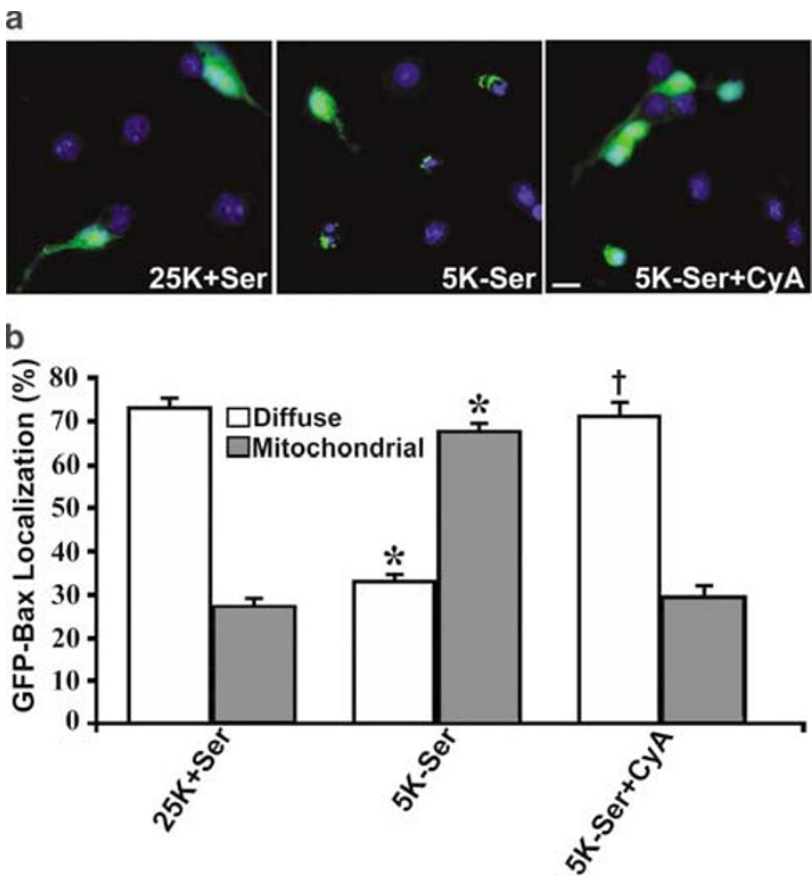

Figure 3 Inhibition of PTP opening with CyA blocks translocation of GFP-Bax to mitochondria. (a) CGNs were transfected with GFP-Bax using a Helios gene gun as described in Materials and Methods and were then incubated for $48 \mathrm{~h}$ to allow for sufficient GFP-Bax expression. CGNs were then incubated for an additional $4 \mathrm{~h}$ in either control ( $25 \mathrm{~K}+$ Ser) or apoptotic $(5 \mathrm{~K}-\mathrm{Ser})$ medium in the absence or presence of CyA $(10 \mu \mathrm{M})$. CGNs were fixed in paraformaldehyde and Hoechst dye was added to stain the nuclei. Images were captured using a $\times 63$ oil immersion lens. GFP-Bax was captured in the FITC channel (shown in green) and Hoechst was captured in the DAPI channel (shown in blue). The images shown are representative of results obtained in three separate experiments. Scale bar, $10 \mu \mathrm{m}$. (b) Quantification of the percentage of cells demonstrating either diffuse or mitochondrial (punctate) GFP-Bax localization was performed for the conditions described in (a). Approximately 75-100 cells/coverslip expressed the GFP-Bax. The percentage of diffuse versus mitochondrial GFP-Bax-positive cells per incubation condition is shown as the mean+S.E.M. of three independent experiments, each performed in duplicate. *Significantly different from the $25 \mathrm{~K}+$ Ser GFP-Bax distribution $(P<0.01)$. ${ }^{\dagger}$ Significantly different from the $5 \mathrm{~K}-$ Ser GFP-Bax distribution $(P<0.01)$ tion of the fusion protein. The fraction of CGNs displaying diffuse GFP-Bax decreased significantly $(P<0.01)$ to $33 \pm 2 \%$ in apoptotic conditions, with a corresponding increase in mitochondrial localization. Inclusion of CyA, at the time of trophic factor deprivation, sustained the diffuse GFP-Bax fraction at $67 \pm 3 \%$ of the GFP-Bax-expressing CGNs. Similar to its lack of effect on MPTP opening, FK506 had no effect on the translocation of GFP-Bax to mitochondria observed in apoptotic medium (data not shown). These results suggest that opening of the MPTP acts as an initiating stimulus to trigger Bax translocation to mitochondria in trophic factordeprived CGNs.

\section{Bax T182A constitutively localizes to mitochondria in human embryonic kidney 293 cells}

Translocation of Bax from the cytosol to the mitochondria is critical for initiation of the intrinsic apoptotic cascade. ${ }^{30}$ Once localized to mitochondria, Bax oligomerizes to form a pore in the outer mitochondrial membrane that is permeable to cytochrome $c .^{31,32}$ To further define the role of the MPTP in Bax localization, we created a novel mutant of $\operatorname{Bax} \alpha$ that constitutively localizes to mitochondria even in the absence of an apoptotic stimulus. This mutant contains a threonine 182 to alanine substitution that is thought to expose the C-terminal helix of Bax for membrane insertion, similar to that described for an S184A mutant. ${ }^{33}$ We transfected GFP-Bax T182A into human embryonic kidney (HEK) 293 cells and compared its localization to wild-type (WT) Bax. GFP-Bax WT showed a diffuse distribution in transfected HEK293 cells (Figure 4a, left panel), whereas GFP-Bax T182A displayed a punctate staining pattern associated with mitochondria (Figure $4 \mathrm{a}$, right panel). To confirm the differential localization of WT and mutant Bax, we isolated cytosolic and mitochondrial fractions from HEK293 cells transfected with either GFP-Bax WT or GFP-Bax T182A. Immunoblotting with an antibody to GFP revealed that cells transfected with GFP-Bax WT contained approximately equal amounts of GFP-Bax in cytosolic and mitochondrial fractions (Figure $4 \mathrm{~b}$, first and second lanes). In contrast, cells transfected with GFP-Bax T182A expressed mutant GFP-Bax that was almost entirely localized in the mitochondrial fraction (Figure $4 \mathrm{~b}$, third and fourth lanes). The integrity of the fractionation procedure was confirmed by reprobing the Western blot for a mitochondrial marker, COX IV (Figure 4b, lower blot).

\section{Bax T182A causes cytochrome $c$ release, caspase activation, and apoptosis in HEK293 cells}

Although we showed that GFP-Bax T182A constitutively localized to mitochondria, it was important to determine if this movement induced apoptosis via the intrinsic cascade. To accomplish this, HEK293 cells were transfected with GFP. Bax T182A and immunostained with primary antibodies recognizing cytochrome $c$, and active (cleaved) fragments of caspase-9 and -3 . Healthy HEK293 cells not expressing the GFP-Bax T182A mutant (cells in field not indicated by arrows) displayed intact nuclei and cytochrome $c$ staining that was mainly associated with mitochondria (Figure 5a). In contrast, 

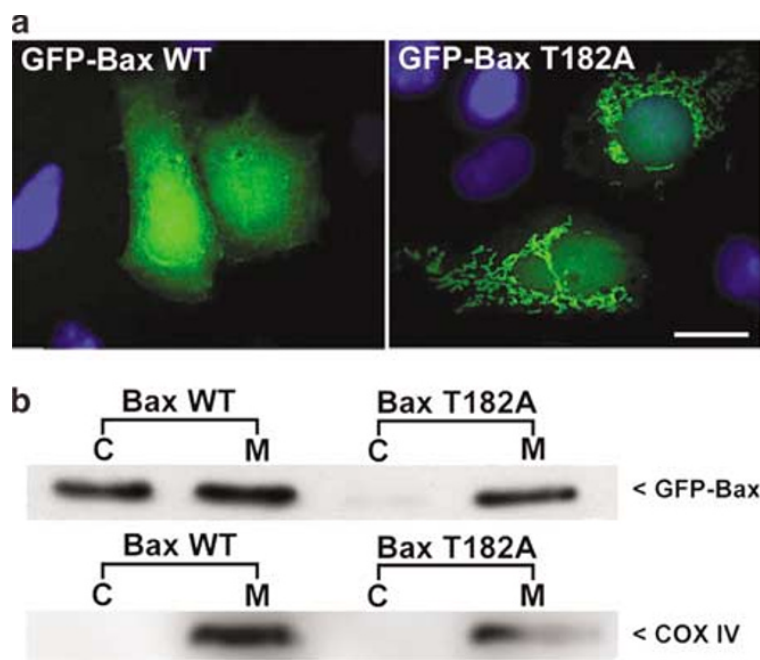

Figure 4 GFP-Bax T182A transiently expressed in HEK293 cells is constitutively localized to mitochondria. (a) HEK293 cells were transfected with either GFP-Bax WT or GFP-Bax T182A using the lipofectamine method. Cells were incubated for $24 \mathrm{~h}$ after transfection, fixed, incubated with Hoechst dye, and visualized for GFP-Bax localization. GFP-Bax WT-expressing cells showed a diffuse staining of the fusion protein throughout most of the cell. In contrast, GFPBax T182A-expressing HEK cells showed extremely punctate staining displayed by concentrated fluorescence within mitochondria. Cells were imaged in $2 \mathrm{ml}$ of PBS with $a \times 63$ water lens. (b) Confirmation of GFP-Bax T182A localization to mitochondria was completed by fractionation of HEK293 cells expressing either GFP-Bax WT or GFP-Bax T182A. Cells were lysed and fractionated to separate the mitochondrial fraction (M) from the cytosolic fraction $(C)$ as described in Materials and Methods. Fractions of each were run on $15 \%$ SDS-polyacrylamide gels, transferred to PVDF membranes, and immunoblotted for GFP using a monoclonal antibody to detect GFP-Bax. The GFP-Bax blot confirmed that cells transfected with GFP-Bax WT display an approximately equal distribution of Bax in cytosol and mitochondria, whereas cells transfected with GFP-Bax T182A had almost all of the GFP-Bax located in mitochondria. The blot was stripped and reprobed for COX IV to confirm that the fractions were indeed cytosolic and mitochondrial as indicated

cells expressing the GFP-Bax T182A mutant (indicated by white arrows) were clearly apoptotic as seen by $4^{\prime}, 6-$ diamidino-2-phenylindole (DAPI) staining illustrating condensed and fragmented nuclei in GFP-positive cells (Figure 5a-C). GFP-Bax T182A-expressing cells showed either a diffuse cytochrome $c$ staining pattern, indicative of mitochondria that had lost their membrane integrity, or little to no cytochrome $c$ staining due to late phases of apoptosis where cytochrome $c$ has completely escaped the cell (Figure $5 \mathrm{a}$ ). In addition to cytochrome $c$ release, active caspase- 9 and -3 were detected in GFP-Bax T182A-positive cells (Figure 5b and c). The ability of Bax T182A to induce apoptosis in HEK cells was confirmed by assessing nuclear condensation in cells expressing either Bax WT or Bax T182A. HEK cells expressing GFP-Bax WT displayed a low level of apoptosis $(21 \pm 5 \%)$ that was significantly greater than cells expressing GFP alone. Cells expressing GFP-Bax T182A showed a significant increase in the amount of apoptosis $(46 \pm 7 \%)$, relative to WT Bax-expressing cells (Table 1 ). These data indicate that the constitutive mitochondrial localization of the Bax point mutant, T182A, induces HEK cell apoptosis through cytochrome $c$ release and caspase activation.

\section{The T182A point mutant of GFP-Bax constitutively localizes to mitochondria and induces CGN apoptosis in the absence of MPTP opening}

We have demonstrated the functional result of expressing the GFP-Bax T182A mutant in HEK cells (i.e., induction of mitochondrial apoptosis). To determine if triggering Bax translocation to mitochondria is indeed the major function of mPTP opening during CGN apoptosis, we examined the effects of the Bax T182A point mutant on CGN apoptosis and mitochondrial membrane potential. In agreement with the data in HEK cells, GFP-Bax T182A demonstrated a punctate distribution when expressed in CGNs maintained in control $(25 \mathrm{~K}+$ Ser) medium (Figure 6a). Consistent with its localization to mitochondria, expression of GFP-Bax T182A in CGNs induced the release of cytochrome $c$ (Figure $6 \mathrm{~b}$, upper panels) and activation of the intrinsic initiator caspase-9 (Figure 6b, middle panels) and the executioner caspase-3 (Figure 6b, lower panels). In accordance with activation of the intrinsic death pathway culminating with activation of caspase-3, GFPBax T182A induced CGN apoptosis at a significantly higher rate than was observed with either GFP, a measure of the baseline apoptosis induced following gene gun transfection, or WT GFP-Bax maintained in control medium (Table 1). These results demonstrate that constitutive localization of Bax to mitochondria is sufficient to induce CGN apoptosis even in the presence of trophic support.

Finally, to determine if the apoptosis induced by constitutive localization of GFP-Bax T182A to mitochondria required opening of the MPTP, we measured the mitochondrial membrane potential in CGNs expressing this point mutant. To assess the loss of membrane potential, TMRE, a dye sensitive to intact mitochondrial membrane potential, was utilized. The left panel of Figure 7a illustrates that under healthy conditions $(25 \mathrm{~K}+$ Ser) CGNs contain abundant TMRE staining, but the right panel shows that apoptotic conditions (5K-Ser) cause CGNs to have almost no TMRE staining indicative of MPTP opening. After $48 \mathrm{~h}$ of incubation in control medium post-transfection, transfected CGNs showed clear mitochondrial localization of GFP-Bax T182A as well as condensed and fragmented chromatin, consistent with induction of apoptosis (Figure 7b, left panel). However, even while undergoing apoptosis, transfected CGNs demonstrated an intact mitochondrial membrane potential as shown by a significant accumulation of TMRE (Figure $7 \mathrm{~b}$, right panel). These data show that constitutive localization of Bax to mitochondria does not by itself trigger mPTP opening, but still induces apoptosis (Figure 7c, middle bar). To further strengthen this argument, we have counted CGNs expressing GFP-Bax T182A that have mitochondrial GFP fluorescence as well as mitochondrial TMRE fluorescence. Of the cells that were GFP-positive, approximately $78 \pm 3.8 \%$ showed mitochondrial localization. Of these $78 \pm 3.8 \%$, approximately $95 \pm 1.2 \%$ had mitochondrial TMRE fluorescence. Moreover, inclusion of CyA throughout the $48 \mathrm{~h}$ incubation period had no effect on GFP-Bax T182A localization to mitochondria (data not shown) or its ability to induce apoptosis (Figure 7c, right bar). In support of this, we have previously shown that an isoform of $\mathrm{Bax}, \mathrm{Bax} \sigma$, which lacks 12 aa near the $\mathrm{C}$-terminus of the protein, does not move to mitochondria even in 

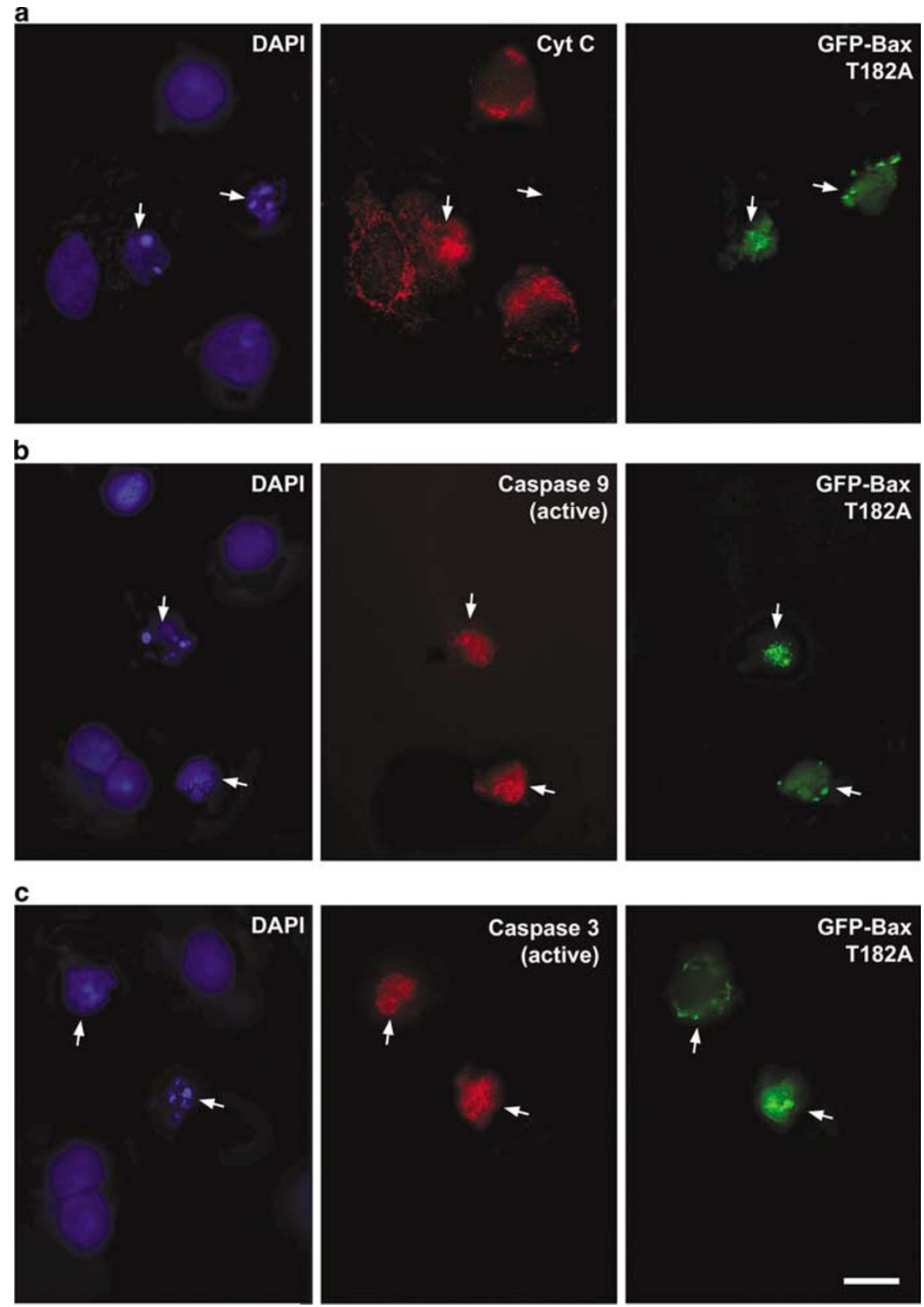

Figure 5 Expression of GFP-Bax T182A in HEK293 cells induces apoptosis by cytochrome $c$ release and activation of caspase-9 and -3 . HEK293 cells were transfected with GFP-Bax T182A as described in Figure 3 legend. The arrows indicate cells expressing GFP-Bax T182A. Cells were allowed to express GFP-Bax T182A for $24 \mathrm{~h}$, and they were then fixed with $4 \%$ paraformaldehyde, permeabilized with $0.2 \%$ Triton X-100, and immunostained as described in Materials and Methods. (a) Cytochrome $c$ release was observed using a rabbit polyclonal primary antibody and a rhodamine-conjugated secondary (red staining). In cells expressing GFP-Bax T182A, cytochrome $c$ was either diffuse (cytosolic) or had permanently left the cell typical of apoptosis induced by the intrinsic death pathway. Cells not expressing GFPBax T182A showed punctate cytochrome $c$ staining within mitochondria. (b) Caspase-9 activation is shown in cells expressing GFP-Bax T182A using a polyclonal antibody that specifically detects cleaved caspase-9. (c) Caspase-3 activation is shown in cells expressing GFP-Bax T182A using a polyclonal antibody that specifically detects cleaved caspase-3. Untransfected cells not expressing GFP-Bax T182A contain no active caspases (absence of staining). Cells were imaged in $2 \mathrm{ml}$ of PBS with $a \times 63$ water lens. All panels in Figure $4 a-c$ are composites of multiple fields captured from two separate experiments

response to trophic factor withdrawal and $\mathrm{MPTP}$ opening (data not shown). ${ }^{42}$ Collectively, these data indicate that the principal role of $\mathrm{MPTP}$ opening in CGN apoptosis is to trigger Bax translocation to mitochondria. Further, by bypassing opening of the MPTP with a Bax mutant that constitutively localizes to mitochondria, CGN apoptosis can be induced in the absence of a permeability transition and is therefore unaffected by CyA.

\section{Discussion}

In this study, we examined the hypothesis that the MPTP plays a role in signaling Bax translocation from the cytosol to mitochondria during neuronal intrinsic apoptosis. We showed that CGNs, deprived of trophic support, commit to intrinsic apoptosis that can be blocked by the addition of CyA. We also showed that Bax moves to the mitochondria following trophic 
Table 1 GFP-Bax T182A induces apoptosis in HEK293 cells and CGNs

\begin{tabular}{lcc}
\hline & \multicolumn{2}{c}{$\%$ Apoptosis } \\
\cline { 2 - 3 } Constructs & HEKs $(\boldsymbol{n}=\mathbf{4})$ & CGNs $(\boldsymbol{n}=\mathbf{6})$ \\
\hline GFP & $1.9 \pm 0.2$ & $9.4 \pm 1.7$ \\
GFP-Bax WT & $21.4 \pm 5.2^{*}$ & $21.5 \pm 2.5^{\star}$ \\
GFP-Bax T182A & $46.7 \pm 7.5^{*, \dagger}$ & $81.4 \pm 2.5^{\star, \dagger}$
\end{tabular}

HEK293 cells were transfected with GFP-Bax T182A as previously described Cells were then incubated in Hoechst dye for $30 \mathrm{~min}$, scraped, centrifuged for $2 \mathrm{~min}$ at $14 \mathrm{kr}$.p.m. to pellet all cells, washed with PBS, recentrifuged, and resuspended in 200-300 $\mu$ l of PBS. A $10 \mu \mathrm{l}$ portion of the cell suspension was then used to count four fields for each condition (GFP; GFP-Bax WT; GFP-Bax T182A) on an Olympus inverted stereomicroscope with a $\times 40$ objective. GFPexpressing cells were counted as apoptotic if they had condensed nuclei and counts are reported as \% apoptosis of total cells expressing GFP. The percentage of GFP-, GFP-Bax-, and GFP-Bax T182A-expressing cells that were apoptotic is shown as the mean \pm S.E.M. of three independen experiments. Quantification of the percent apoptosis of CGNs transfected with GFP, GFP-Bax WT, or GFP-Bax T182A was assessed after 48 h. Approximately 75-100 cells/coverslip expressed GFP, GFP-Bax WT, or GFP-Bax T182A. Each expressing cell was classified as either healthy or apoptotic using nuclear condensation and fragmentation as indicators of apoptosis. Experiments were carried out as above, except that each was performed in duplicate. *Significantly different from the GFP (baseline transfection) control $(P<0.01)$ Significantly different from the GFP-Bax WT $(P<0.01)$

factor withdrawal and this translocation is sensitive to CyA as well. To further support the hypothesis that the MPTP has a role in directing Bax movement, we showed that a Bax T182A mutant localized to mitochondria and induced apoptosis, regardless of the presence of CyA. The data presented here suggest a model for the intrinsic apoptotic pathway in CGNs that involves the $\mathrm{mPTP}$ signaling Bax to translocate to mitochondria and release cytochrome $c$.

It has been previously demonstrated that overexpression of Bax can lead to apoptosis in the absence of an internal or external apoptotic stimulus. ${ }^{34}$ It is possible that in our model, overexpression of the Bax T182A mutant induces apoptosis by a mechanism separate from that of trophic factor withdrawal. Although these two activators of apoptosis may initiate death differently, we have shown that they converge on a common, intrinsic pathway that involves cytochrome $c$ release and caspase- 9 and -3 activation, and is sensitive to CyA.

Previous studies examining the relationship between the mPTP and Bax in apoptosis have produced contradictory results. Overall, this debate stems from three perspectives of how the intrinsic mitochondrial apoptotic cascade is initiated. First, Bax alone may be sufficient to induce cytochrome $c$ release without opening of the $\mathrm{mPTP} .{ }^{35}$ Second, opening of the MPTP alone may be enough to induce cytochrome $c$ release without Bax translocation, oligomerization, or insertion. ${ }^{36}$ And third, it has been proposed that Bax may form a composite pore with the $\mathrm{MPTP}$ that is necessary to release cytochrome $c .^{37}$ In vitro studies have shown that recombinant Bax can induce cytochrome $c$ release from isolated mitochondria, independent of the cell type specific origin, with or without opening of the MPTP. ${ }^{35}$ However, the data presented here confirm the requirement for Bax in neuronal apoptosis and indicate that the mPTP must signal to Bax to induce its translocation to mitochondria.

A Bax-only pore model cannot explain results showing that Bax interacts with a putative component of the MPTP, the

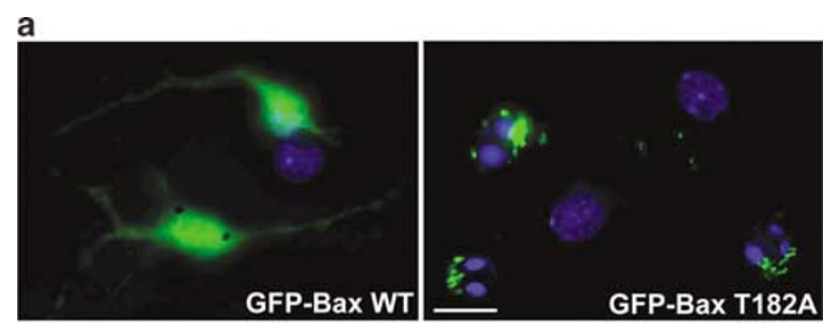

b

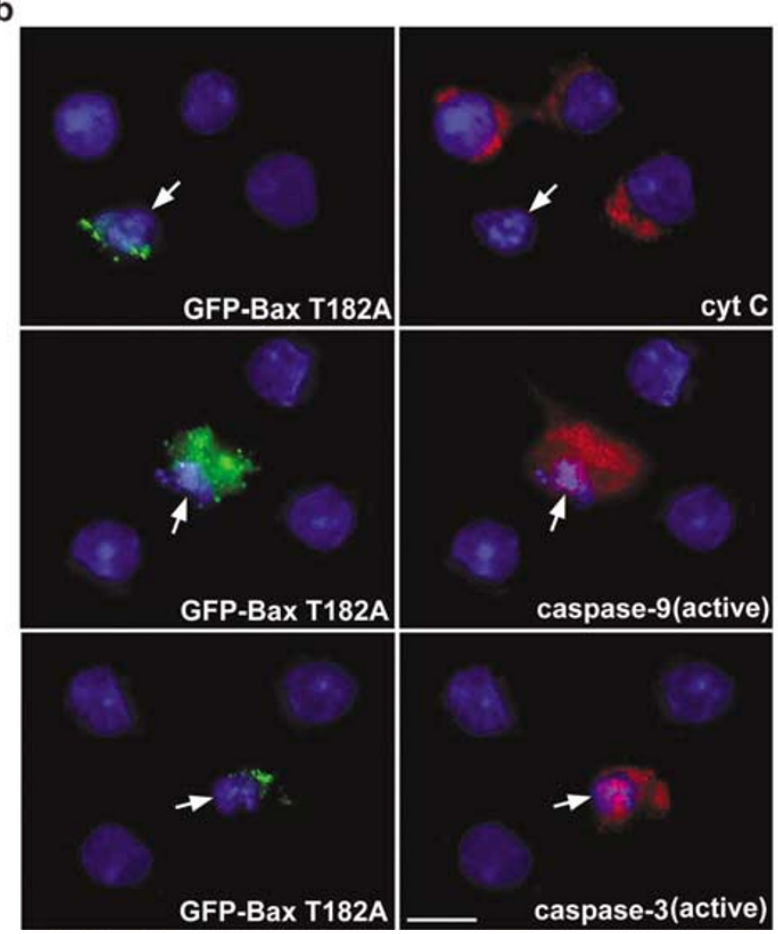

Figure 6 GFP-Bax T182A constitutively localizes to mitochondria in CGNs and induces cytochrome $c$ release and apoptosis. (a) CGNs were transfected with either GFP-Bax WT or GFP-Bax T182A using a Helios gene gun as described in Materials and Methods and were incubated in control $(25 \mathrm{~K}+\mathrm{Ser})$ medium for $48 \mathrm{~h}$ to allow for sufficient GFP-Bax expression. Cells were fixed in paraformaldehyde and Hoechst dye was added to stain the nuclei. Images were captured using a $63 \times$ oil immersion lens. GFP-Bax was captured in the FITC channel (shown in green) and Hoechst was captured in the DAPI channel (shown in blue). Note that the mutant Bax constitutively localized to the mitochondria even in control medium, while the WT GFP-Bax demonstrated primarily diffuse staining. The images shown are representative of results obtained in four separate experiments, each performed in duplicate. Scale bar, $10 \mu \mathrm{m}$. (b) CGNs were transfected with GFP-Bax T182A using a Helios gene gun as described in Materials and Methods and allowed to incubate in control $(25 \mathrm{~K}+\mathrm{Ser})$ medium for $48 \mathrm{~h}$ to allow for sufficient GFP-Bax T182A expression. After incubation, the CGNs were fixed in 4\% paraformaldehyde, permeabilized with $0.2 \%$ Triton X-100, and blocked with $5 \%$ BSA. Cytochrome $c$ (cyt c), active caspase-9, and active caspase- 3 were immunostained by incubating the cells with polyclonal antibodies to cyt $c$ and the active fragments of caspase- 9 and -3 , followed by Cy3-conjugated secondary antibodies. Fluorescent images were captured using a $\times 100$ oil objective. Note that both caspase- 3 and -9 were active only in the cells expressing GFP-Bax T182A and that the cyt $c$ was released by the mitochondria only in cells expressing the mutant Bax. The images shown are representative of results obtained in three separate experiments, each performed in duplicate. Scale bar, $10 \mu \mathrm{m}$

VDAC. ${ }^{38}$ Furthermore, Bax has been shown to induce opening of a VDAC channel in synthetic liposomes and this opening can be blocked by CyA. ${ }^{39,40}$ Given the Bax-VDAC 

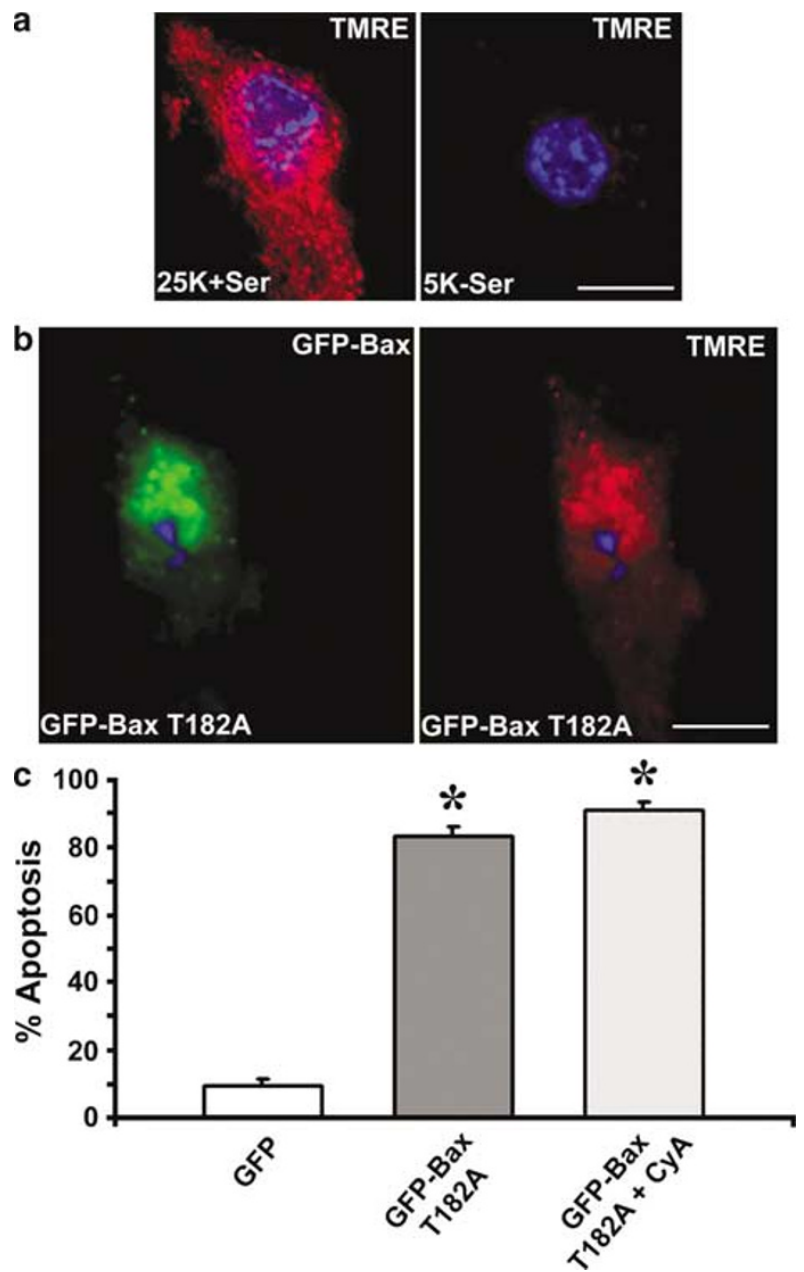

Figure 7 Constitutive localization of GFP-Bax T182A to mitochondria induces CGN apoptosis independently of MPTP opening. (a) Rat CGNs were treated and imaged exactly as described in Figure $1 \mathrm{~b}$ with regard to cells stained with TMRE. After incubation with Hoechst dye and TMRE $(500 \mathrm{nM})$, the coverslips were inverted onto slides into a small volume of PBS containing glucose and $p$ phenylenediamine (antiquenching agent). Living cells were then were imaged under a $\times 100$ oil lens. Under healthy conditions $(25 \mathrm{~K}+\mathrm{Ser})$, cells have distinct, localized staining of TMRE displaying mitochondria with intact membrane potentials. Under apoptotic conditions $(5 \mathrm{~K}-\mathrm{Ser})$, cells show little to no TMRE staining, indicating that the MPTP has formed and mitochondrial membranes have lost their potential. (b) Rat CGNs were transfected with GFP-Bax T182A using the Helios gene gun and cells were maintained in control medium (25K + Ser). The left panel shows a cell expressing GFP-Bax T182A, identified by GFP fluorescence, imaged using the FITC channel (green fluorescence), where Bax T182A is constitutively localized to mitochondria, and as a result the cell is undergoing apoptosis. In the right panel, the same cell is stained by TMRE (red fluorescence) in a punctate distribution that colocalized with the GFP-Bax fluorescence, indicating that while the GFP-Bax is constitutively localized to mitochondria and apoptosis is induced by this localization, mitochondrial membranes still maintain their potential. (c) Apoptosis induced by the constitutively mitochondrial GFP-Bax T182A cannot be blocked by the addition of cyclosporin A Rat CGNs were transfected with either GFP or GFP-Bax T182A as previously described. They were allowed to incubate in control medium $(25 \mathrm{~K}+\mathrm{Ser})$ for $48 \mathrm{~h}$ in the presence or absence of CyA $(10 \mu \mathrm{M})$ and Hoechst dye was added to stain the nuclei. Imaging for counting was performed using a $63 \times$ oil immersion lens. Cells were considered apoptotic if their nuclei were either condensed and/or fragmented. Approximately 200 GFP-Bax-expressing cells were counted from three replicate coverslips per experiment, with each experiment performed at least three times. Cells expressing GFP-Bax T182A in the presence or absence of CyA that were scored as apoptotic are shown as the mean \pm S.E.M. of three independent experiments. * Significantly different from the GFP (baseline transfection) control $(P<0.01)$ interaction data, a VDAC/cyclophilin D-composed mPTP supports the model that a composite channel is likely to exist; however, in vivo colocalization of any components of the mPTP with Bax has yet to be shown.

It is presently unclear how the PTP opening triggers Bax translocation to the mitochondria. It is known that PTP opening causes shape changes in mitochondria. ${ }^{5}$ It is also known that certain members of the Bcl-2 family, such as Bim, are associated with the dynein motor complex. ${ }^{8}$ It is possible that an association between Bax and dynein may exist and be regulated by mitochondrial shape change. It has also been demonstrated that Bax is sequestered in the cytosol by the scaffolding protein 14-3-3 and that this interaction negatively regulates Bax movement. ${ }^{41}$ This Bax/14-3-3 interaction might be a site for regulation by the MPTP.

In summary, we have found that CyA blocks opening of the mPTP and the release of cytochrome $c$ in CGNs deprived of trophic support, without affecting Bim expression. Moreover, inhibition of the MPTP with CyA efficiently blocked Bax translocation to mitochondria. A T182A mutant of GFP-Bax that constitutively localized to mitochondria induced cytochrome $c$ release, caspase- 9 and -3 activation, and CGN apoptosis without inducing opening of the PTP. Further, the constitutive localization of Bax T182A to mitochondria and its ability to induce CGN apoptosis were unaffected by the addition of CyA. We conclude that the major role of the PTP during neuronal apoptosis induced by trophic factor withdrawal is to direct Bax localization to mitochondria.

\section{Materials and Methods}

\section{Materials}

cDNA encoding for the $\alpha$-isoform of human Bax protein fused to GFP (GFP-Bax) was kindly provided by Dr. Richard Youle (NIH, Bethesda, MD, USA). FK506 and CyA were purchased from Calbiochem (La Jolla, CA, USA). Hoechst dye no. $33258, \mathrm{CoCl}_{2} \cdot 6 \mathrm{H}_{2} \mathrm{O}$, DAPI, and recombinant human IGF-I were obtained from Sigma (St. Louis, MO, USA). CalceinAM, Pluronic F-127, JC-1, and TMRE were obtained from Molecular Probes (Eugene, OR, USA). The Helios gene gun, gold ( $0.6 \mu \mathrm{m}$ diameter) microcarrier beads, and calcium phosphate reagents for plasmid DNA precipitation were obtained from Bio-Rad (Hercules, CA, USA). EGFP plasmid DNA was obtained from BD Biosciences/Clontech (Palo Alto, CA, USA). The GFP monoclonal antibody and the COX IV monoclonal antibody were purchased from Clontech (Palo Alto, CA, USA). The cytochrome $c$ rabbit polyclonal antibody was purchased from Santa Cruz Biotechnology (Santa Cruz, CA, USA). The caspase-3 rabbit polyclonal antibody was purchased from Promega (Madison, WI, USA). The caspase-9 rabbit polyclonal antibody was obtained from Cell Signaling Technology (Beverly, MA, USA). Cy3-conjugated secondary antibodies for immunocytochemistry were purchased from Jackson Immunoresearch Laboratories (West Grove, PA, USA). Horseradish peroxidase-linked secondary antibodies and reagents for enhanced chemiluminescence detection were obtained from Amersham Pharmacia Biotechnologies (Piscataway, NJ, USA).

\section{Site-directed mutagenesis of Bax}

Mutants of human GFP-Bax $\alpha$ were created using a QuickChange sitedirected mutagenesis kit (Stratagene) according the manufacturer's 
recommended procedure. Human GFP-Bax $\alpha$ point mutations were created in the following way: the T182A substitution mutation was obtained with the mutagenic forward primer $5^{\prime}$-GGGAGTGCTCGCCGCC TCACTCACCATCTGG- $3^{\prime}$ and its complementary reverse primer. Primers were supplied by Invitrogen (Carlsbad, CA, USA) and IDT (Coralville, IA, USA).

\section{Rat cerebellar granule neuron cultures}

Rat CGNs were isolated from 7-day-old Sprague-Dawley rat pups (15$19 \mathrm{~g}$ ) as described previously. ${ }^{3}$ Briefly, neurons were plated at a density of $4.0 \times 10^{6}$ cells $/ 35-\mathrm{mm}$ well in basal modified Eagle's medium containing $10 \%$ fetal bovine serum, $25 \mathrm{mM} \mathrm{KCl}, 2 \mathrm{mM}$ L-glutamine, and penicillin $(100 \mathrm{U} / \mathrm{ml})$-streptomycin $(100 \mu \mathrm{g} / \mathrm{ml})$ (Invitrogen, Grand Island, NY, USA). Cytosine arabinoside $(10 \mu \mathrm{M})$ was added to the culture medium $24 \mathrm{~h}$ after plating to limit the growth of non-neuronal cells. Using this protocol, the cultures were $\sim 95 \%$ pure for granule neurons. In general, experiments were performed after 7 days in culture.

\section{Helios gene gun transfection}

Gold ( $0.6 \mu \mathrm{m}$ diameter) microcarrier beads were coated with the desired plasmid DNA encoding for either GFP, GFP-Bax WT, or GFP-Bax T182A mutant using a calcium phosphate precipitation method described by the Helios gene gun manufacturer (Bio-Rad). The gold beads were then coated on the inside of plastic tubing and the tubing was cut into individual cartridges. Helium at a pressure of $100 \mathrm{psi}$ was shot through the tubing to deliver the plasmid-coated beads into the CGN cultures.

\section{HEK293 cell culture and transfection}

HEK293 cells were cultured in Dulbecco's modified Eagle's medium (DMEM) with $10 \%$ fetal calf serum, $10 \mu \mathrm{g} / \mu \mathrm{l}$ of streptomycin, and $10 \mathrm{IU} / \mathrm{ml}$ of penicillin. The cultures were incubated at $37^{\circ} \mathrm{C}$ and in an atmosphere of $5 \% \mathrm{CO}_{2}$. HEK293 cells were transfected with $0.5-2 \mu \mathrm{g}$ of DNA using the lipofectamine method according to the manufacturer's procedure (Invitrogen).

\section{Cell fractionation}

After incubation of CGNs in either control or apoptotic medium $( \pm 10 \mu \mathrm{M}$ CyA), the medium was removed and CGNs were washed once with $2 \mathrm{ml}$ of ice-cold PBS (pH 7.4). Cells were then placed on ice and scraped into cytosolic extraction buffer $(200 \mu \mathrm{l} / 35-\mathrm{mm}$ well) containing $1 \times$ protease inhibitor cocktail, $1 \mathrm{mM}$ DTT, and $4 \mathrm{ml}$ PBS, each components of the BioVision cytochrome $c$ releasing apoptosis assay kit (Alexis Biochemicals). The lysates were then removed from the wells and each sample was subjected to 35 strokes in a dounce homogenizer on ice.

HEK293 cells expressing GFP-Bax WT or GFP-Bax T182A were lysed and treated as previously described for CGNs. Cells were washed with $1 \times$ PBS, incubated with $200 \mu \mathrm{l}$ of cytosol extraction buffer for $20 \mathrm{~min}$, scraped and harvested, and homogenized with a dounce homogenizer.

The lysates from CGNs or HEK cells were centrifuged at $700 \times g$ for $10 \mathrm{~min}$, the supernatant was transferred to a new tube, centrifuged again at $10000 \times g$ for $30 \mathrm{~min}$, and this supernatant was transferred to a new tube labeled cytosolic fraction. The pellet was resuspended in mitochondria extraction buffer provided by Alexis containing DTT (1 M) and protease inhibitors, and then labeled mitochondrial fraction. Fractions were analyzed by SDS-polyacrylamide gel electrophoresis (SDS-PAGE) using $15 \%$ gels and then subjected to Western blotting, probing initially for cytochrome $c$ or GFP-Bax and then reprobing for COX IV (mitochondrial marker).

\section{Immunoblot analysis}

Nonspecific binding sites were blocked in PBS (pH 7.4) containing $0.1 \%$ Tween 20 (PBS-T) and 1\% BSA for $1 \mathrm{~h}$ at room temperature. Primary rabbit polyclonal antibodies were diluted in blocking solution (final dilution of $1: 250-1: 1000)$ and incubated with the membranes for $1 \mathrm{~h}$. Excess primary antibody was removed by washing the membranes three times in PBS-T. The blots were then incubated with horseradish peroxidaseconjugated anti-rabbit secondary antibody diluted in PBS-T (final dilution of $1: 5000$ ) for $1 \mathrm{~h}$ and subsequently washed three times with PBS-T. Immunoreactive proteins were detected by enhanced chemiluminescence. In some experiments, membranes were reprobed after stripping in $0.1 \mathrm{M}$ Tris- $\mathrm{HCl}$ (pH 8.0), $2 \%$ SDS, and $100 \mathrm{mM} \beta$-mercaptoethanol for $30 \mathrm{~min}$ at $52^{\circ} \mathrm{C}$. The blots were rinsed twice in PBS-T and processed as above with a different primary antibody. Autoluminograms shown are representative of at least three different experiments. Densitometry measurements were performed on $\mathrm{Bim}_{\mathrm{s}}$ immunoblots normalizing to $25 \mathrm{~K}+$ Ser and represented as a fold change of density. Data analysis was performed using ANOVA two-factor statistics.

\section{Measurement of mitochondrial swelling}

CGNs were plated onto polyethyleneimine-coated glass coverslips at a density of approximately $2 \times 10^{5}$ cells/coverslip. CGNs were incubated in either control medium or apoptotic medium in the absence or presence of CyA. JC-1 (final concentration of $2 \mu \mathrm{g} / \mathrm{ml}$ ) and Hoechst dye were added directly to the culture medium $30 \mathrm{~min}$ prior to fixation to stain mitochondria and nuclei, respectively. JC-1 fluorescence was captured in paraformaldehyde-fixed cells using a Сy3 filter under $a \times 100$ oil objective.

\section{Assessment of mitochondrial membrane potential}

CGNs plated on glass coverslips were incubated in either control or apoptotic medium $( \pm$ CyA). TMRE (final concentration, $500 \mathrm{nM})$ and Hoechst dye were added directly to the cells $30 \mathrm{~min}$ prior to the end of the incubation period. Following incubation, coverslips were inverted onto slides into a small volume of phenol red-free medium containing TMRE $(500 \mathrm{nM})$. Living cells were then imaged using a Cy3 filter to detect TMRE fluorescence under a $\times 100$ oil objective. All images were acquired at equal exposure times for TMRE fluorescence to assess the relative mitochondrial membrane potentials. Counts of CGNs expressing GFP-Bax T182A with and without intact TMRE fluorescence were performed as described above and at least 100 cells were counted in three experiments.

\section{Calcein $/ \mathrm{Co}^{2+}$ assay for PTP opening}

CGNs plated on glass coverslips were incubated in either control or apoptotic medium $( \pm \mathrm{CyA})$ for $4 \mathrm{~h}$. At $20 \mathrm{~min}$ prior to the end of the incubation, cells were loaded with calcein-AM to a final concentration of $1 \mu \mathrm{M}$ in the presence of $1 \mathrm{mM} \mathrm{CoCl}_{2}, \sim 0.1 \%$ Pluronic $\mathrm{F}-127$, and Hoechst dye. After the 20 min incubation, calcein-AM cells were washed four times with ice-cold $1 \times$ PBS. Coverslips were inverted onto slides in a small volume of phenol red-free medium containing glucose and loosely mounted. Living cells were imaged immediately using a FITC filter to detect calcein fluorescence under a $\times 100$ oil objective. Calcein fluorescence was confirmed to be mitochondrial by colocalizing with TMRE (final concentration, $500 \mathrm{nM}$ ). 


\section{Preparation of CGN cell extracts}

CGNs were incubated in either control or apoptotic medium $( \pm$ CyA). The culture medium was aspirated, CGNs were washed once with $2 \mathrm{ml}$ of icecold PBS ( $\mathrm{pH} 7.4)$, and cells were then placed on ice and scraped into lysis buffer $(200 \mu \mathrm{l} / 35-\mathrm{mm}$ well) containing $20 \mathrm{mM}$ HEPES $(\mathrm{pH} 7.4), 1 \%$ Triton $\mathrm{X}-100,50 \mathrm{mM} \mathrm{NaCl}, 1 \mathrm{mM}$ EGTA, $5 \mathrm{mM} \beta$-glycerophosphate, $30 \mathrm{mM}$ sodium pyrophosphate, $100 \mu \mathrm{M}$ sodium orthovanadate, $1 \mathrm{mM}$ phenylmethylsulfonyl fluoride, $10 \mu \mathrm{g} / \mathrm{ml}$ leupeptin, and $10 \mu \mathrm{g} / \mathrm{ml}$ aprotinin. Cell debris was removed by centrifugation at $6000 \times g$ for $3 \mathrm{~min}$ and the protein concentration of the supernatant was determined using a commercially available protein assay kit (Pierce Chemical Co., Rockford, IL, USA). Aliquots ( $\sim 150 \mu \mathrm{g}$ ) of supernatant protein were diluted to a final concentration of $1 \times$ SDS-PAGE sample buffer, boiled for $5 \mathrm{~min}$, and electrophoresed through $15 \%$ polyacrylamide gels. Proteins were transferred to polyvinylidene difluoride membranes (Millipore Corp., Bedford, MA, USA) and processed for immunoblot analysis.

\section{Quantification of GFP-Bax localization}

At $48 \mathrm{~h}$ after Helios gene gun transfection, approximately $2 \%$ of the CGNs expressed the transgene. Cells were then subjected to trophic factor withdrawal for $4 \mathrm{~h}$, followed by Hoechst staining and fixation in paraformaldehyde. Two distinct patterns of GFP-Bax expression were observed - diffuse and punctate (mitochondrial). The number of GFP-Baxexpressing cells demonstrating either a diffuse or mitochondrial localization was assessed by counting 50-100 transfected cells per coverslip. Three replicate coverslips were scored per treatment group in a given experiment and each experiment was performed at least three times. The images shown are representative of results obtained from at least three independent experiments.

\section{Quantification of CGN apoptosis}

Apoptosis was induced either by removing serum and decreasing the extracellular potassium concentration from 25 to $5 \mathrm{mM}$ or by transfecting CGNs with the GFP-Bax T182A point mutant followed by incubation for $48 \mathrm{~h}$ in control medium. After the induction of apoptosis, CGNs were fixed with $4 \%$ paraformaldehyde and nuclei were stained with Hoechst dye. Cells were considered apoptotic if their nuclei were either condensed or fragmented. In general, approximately 500 cells from at least two fields of a 35-mm well were counted. For transfection experiments, approximately 200 GFP-Bax (WT or mutant)-expressing cells were counted from three replicate coverslips per experiment, with each experiment performed at least three times. Data are presented as the percentage of cells in a given treatment group that were scored as apoptotic.

\section{Quantification of HEK293 apoptosis}

HEK293 cells were transfected with GFP-Bax WT or GFP-Bax T182A. Hoechst dye was added to each $35 \mathrm{~mm}$ well of HEK cells $(1 \mu \mathrm{l} / 1 \mathrm{ml}$ of media) and allowed to incubate for $20 \mathrm{~min}$. Cells were scraped off the dish, centrifuged, and resuspended in $1 \times$ PBS. Cells were washed again with $1 \times$ PBS, resuspended again in PBS, and applied to microscope slides. Counting was carried out on an Olympus phase contrast inverted microscope using epi-illuminescence at $\times 40$ magnification. Cells were scored as apoptotic if their nuclei were condensed or fragmented, and expression of Bax was confirmed by GFP fluorescence. HEK293 apoptosis was quantified using two replicate dishes per experiment, with each experiment performed at least three times. Data represent the percentage of cells in a given experiment that were scored as apoptotic.

\section{Immunocytochemistry}

HEK293 cells used for visualizing cytochrome $c$ release and caspase activation were fixed in $4 \%$ paraformaldehyde. Cells were washed once with PBS ( $\mathrm{pH} \mathrm{7.4)}$ then permeabilized and blocked with PBS containing $5 \%$ BSA and $0.2 \%$ Triton X-100 for $1 \mathrm{~h}$. Cells were washed once with PBS $(\mathrm{pH} 7.4)$ and allowed to incubate at room temperature with either the rabbit anti-cytochrome $c$, rabbit anti-caspase-3, or rabbit anti-caspase- 9 primary antibodies $(1: 500)$ for $1 \mathrm{~h}$, each diluted in PBS containing $0.2 \%$ Triton $\mathrm{X}$ 100 and $2 \%$ BSA. Primary antibodies were aspirated and the cells were washed five times with PBS. Cells were then incubated at room temperature for $1 \mathrm{~h}$ with the appropriate rhodamine-conjugated or fluorescein isothiocyanate-conjugated secondary antibody $(1: 500)$ and DAPI. Cells were imaged in $2 \mathrm{ml}$ of PBS and captured with a Zeiss Axioplan 2 digital microscope equipped with a Cooke Sensicam deepcooled CCD camera. Images were analyzed and subjected to digital deconvolution using the Slidebook software program (Intelligent Imaging Innovations Inc., Denver, CO, USA). Rat CGNs were treated exactly as described above, with the following exceptions. CGNs were cultured on polyethyleneimine-coated glass coverslips at a density of approximately $2 \times 10^{5}$ cells/coverslip. Cells were then incubated for approximately $16 \mathrm{~h}$ at $4{ }^{\circ} \mathrm{C}$ with primary rabbit polyclonal antibodies to either cytochrome $\mathrm{C}$, active caspase- 9 , or active caspase-3, each diluted in PBS containing $0.2 \%$ Triton X-100 and $2 \%$ BSA. After addition of secondary antibody, CGNs were then washed five more times with PBS, and coverslips were adhered to glass slides in mounting medium $(0.1 \%$ p-phenylenediamine in $75 \%$ glycerol in PBS). Fluorescent images were captured using a $\times 63$ oil immersion objective.

\section{References}

1. Honig LS and Rosenberg RN (2000) Apoptosis and neurologic disease. Am. J. Med. 4: 317-330

2. Friedlander RM and Yuan $J$ (1998) ICE, neuronal apoptosis and neurodegeneration. Cell Death Differ. 10: 823-831

3. D'Mello SR, Galli C, Ciotti T and Calissano P (1993) Induction of apoptosis in cerebellar granule neurons by low potassium: inhibition of death by insulin-like growth factor I and cAMP. Proc. Natl. Acad. Sci. USA 23: 10989-10993

4. Gerhardt E, Kugler S, Leist M, Beier C, Berliocchi L, Volbracht C, Weller M, Bahr M, Nicotera P and Schulz JB (2001) Cascade of caspase activation in potassium-deprived cerebellar granule neurons: targets for treatment with peptide and protein inhibitors of apoptosis. Mol. Cell. Neurosci. 4: 717-731

5. Linseman DA, Phelps RA, Bouchard RJ, Le SS, Laessig TA, McClure ML and Heidenreich KA (2002) Insulin-like growth factor-I blocks Bcl-2 interacting mediator of cell death (Bim) induction and intrinsic death signaling in cerebellar granule neurons. J. Neurosci. 21: 9287-9297

6. Korsmeyer SJ, Shutter JR, Veis DJ, Merry DE and Oltvai ZN (1993) Bcl-2/Bax: a rheostat that regulates an anti-oxidant pathway and cell death. Semin. Cancer Biol. 6: 327-332

7. Tsujimoto $Y$ (1998) Role of Bcl-2 family proteins in apoptosis: apoptosomes or mitochondria? Genes Cells 11: 697-707

8. Puthalakath H, Huang DC, O'Reilly LA, King SM and Strasser A (1999) The proapoptotic activity of the Bcl-2 family member Bim is regulated by interaction with the dynein motor complex. Mol. Cell 3: 287-296

9. Letai A, Bassik MC, Walensky LD, Sorcinelli MD, Weiler S and Korsmeyer SJ (2002) Distinct BH3 domains either sensitize or activate mitochondrial apoptosis, serving as prototype cancer therapeutics. Cancer Cell 3: 183-192 
10. Marani M, Tenev T, Hancock D, Downward J and Lemoine NR (2002) Identification of novel isoforms of the $\mathrm{BH} 3$ domain protein Bim which directly activate Bax to trigger apoptosis. Mol. Cell. Biol. 11: 3577-3589

11. Korsmeyer SJ (1995) Regulators of cell death. Trends Genet. 3: 101-105

12. Hirsch T, Marzo I and Kroemer G (1997) Role of the mitochondrial permeability transition pore in apoptosis. Biosci. Rep. 1: 67-76

13. Martinou I, Desagher S, Eskes R, Antonsson B, Andre E, Fakan S and Martinou JC (1999) The release of cytochrome $c$ from mitochondria during apoptosis of NGF-deprived sympathetic neurons is a reversible event. J. Cell Biol. 5: 883-889

14. Kokozka JE, Waymire KG, Levy SE, Sligh JE, Cai J, Jones DP, Macgregor GR and Wallace DC (2004) The ADP/ATP translocator is not essential for the mitochondrial permeability transition pore. Nature 6973: 461-465

15. Zamzami N and Kroemer $\mathrm{G}$ (2001) The mitochondrion in apoptosis: how Pandora's box opens. Nat. Rev. Mol. Cell Biol. 1: 67-71

16. De Giorgi F, Latigue L, Bauer MK, Schubert A, Grimm S, Hanson GT, Remington SJ, Youle RJ and Ichas F (2002) The permeability transition pore signals apoptosis by directing Bax translocation and multimerization. FASEB J. 6: 607-609

17. Ikemoto H, Tani E, Ozaki I, Kitagawa H and Arita N (2000) Calphostin Cmediated translocation and integration of Bax into mitochondria induces cytochrome $c$ release before mitochondrial dysfunction. Cell Death Differ. 6 : $511-520$

18. Krohn AJ, Wahlbrink T and Prehn JH (1999) Mitochondrial depolarization is not required for neuronal apoptosis. J. Neurosci. 17: 7394-7404

19. Crompton M (1999) The mitochondrial permeability transition pore and its role in cell death. Biochem. J. 341: 233-249

20. Petronilli V, Miotto G, Canton M, Brini M, Colonna R, Bernardi P and Di Lisa F (1999) Transient and long-lasting openings of the mitochondrial permeability transition pore can be monitored directly in intact cells by changes in mitochondrial calcein fluorescence. Biophys. J. 2: 725-734

21. Uchino H, Minamikawa-Tachino R, Kristian T, Perkins G, Narazaki M, Siesjo BK and Shibasaki $F$ (2002) Differential neuroprotection by cyclosporin $A$ and FK506 following ischemia corresponds with differing abilities to inhibit calcineurin and the mitochondrial permeability transition. Neurobiol. Dis. 3: 219-233

22. Von Ahsen O, Waterhouse NJ, Kuwana T, Newmeyer DD and Green DR (2000) The 'harmless' release of cytochrome c. Cell Death Differ. 12: 11921199

23. Zou H, Li Y, Liu X and Wang X (1999) An APAF-1.cytochrome $c$ multimeric complex is a functional apoptosome that activates procaspase-9. J. Biol. Chem. 17: $11549-11556$

24. Li P, Nijhawan D, Budihardjo I, Srinivasula SM, Ahmad M, Alnemri M and Wang $X$ (1997) Cytochrome $c$ and dATP-dependent formation of Apaf-1/caspase- 9 complex initiates an apoptotic protease cascade. Cell 4: 479-489

25. Putcha GV, Moulder KL, Golden JP, Bouillet P, Adams JA, Strasser A and Johnson EM (2001) Induction of BIM, a proapoptotic BH3-only BCL-2 family member, is critical for neuronal apoptosis. Neuron 3: 615-628

26. Whitfield J, Neame SJ, Paquet L, Bernard O and Ham J (2001) Dominantnegative c-Jun promotes neuronal survival by reducing BIM expression and inhibiting mitochondrial cytochrome $c$ release. Neuron 3: 629-643

27. D'Mello SR, Borodezt K and Soltoff SP (1997) Insulin-like growth factor and potassium depolarization maintain neuronal survival by distinct pathways: possible involvement of PI 3-kinase in IGF-1 signaling. J. Neurosci. 5: 1548-1560

28. Chrysis D, Calikoglu AS, Ye P and D'Ercole AJ (2001) Insulin-like growth factorI overexpression attenuates cerebellar apoptosis by altering the expression of Bcl family proteins in a developmentally specific manner. J. Neurosci. 5: 14811489

29. Becker DJ and MacDougald OA (1999) Transfection of adipocytes by gene gun-mediated transfer. Biotechniques 4: 660-662, 668

30. Wolter KG, Hsu YT, Smith CL, Nechushtan A, Xi XG and Youle RJ (1997) Movement of Bax from the cytosol to mitochondria during apoptosis. J. Cell Biol. 5: 1281-1292

31. Antonsson B, Montessuit S, Lauper S, Eskes R and Martinou JC (2000) Bax oligomerization is required for channel-forming activity in liposomes and to trigger cytochrome $c$ release from mitochondria. Biochem. J. 345: 271-278

32. Korsmeyer SJ, Wei MC, Saito M, Weiler S, On KJ and Schlesinger PH (2000) Pro-apoptotic cascade activates BID, which oligomerizes BAK or BAX into pores that result in the release of cytochrome $c$. Cell Death Differ. 12: 11661173

33. Nechushtan A, Smith CL, Hsu YT and Youle RJ (1999) Conformation of the Bax C-terminus regulates subcellular location and cell death. EMBO J. 9: 2330-2341

34. Kitanaka C, Namiki T, Noguchi K, Mochizuki T, Kagaya S, Chi S, Hayashi A, Asai A, Tsujimoto $Y$ and Kuchino $Y$ (1997) Caspase-dependent apoptosis of COS-7 cells induced by Bax overexpression: differential effects of Bcl-2 and $\mathrm{BCl}-\mathrm{xL}$ on Bax-induced caspase activation and apoptosis. Oncogene 15: 17631772

35. Jurgensmeier JM, Xie Z, Deveraux Q, Ellerby L, Bredesen D and Reed JC (1998) Bax directly induces release of cytochrome $c$ from isolated mitochondria. Proc. Natl. Acad. Sci. USA 9: 4997-5002

36. Petit PX, Goubem M, Diolez P, Susin SA, Zamzami N and Kroemer G (1998) Disruption of the outer mitochondrial membrane as a result of large amplitude swelling: the impact of irreversible permeability transition. FEBS Lett. 1: 111-116

37. Marzo I, Brenner C, Zamzami N, Jurgensmeier JM, Susin SA, Vieira HL, Prevost MC, Xie Z, Matsuyama S, Reed JC and Kroemer G (1998) Bax and adenine nucleotide translocator cooperate in the mitochondrial control of apoptosis. Science 5385: 2027-2031

38. Shimizu S, Ide T, Yanagida T and Tsujimoto Y (2000) Electrophysiological study of a novel large pore formed by Bax and the voltage-dependent anion channel that is permeable to cytochrome $c$. J. Biol. Chem. 16: 12321-12325

39. Shimizu S, Narita M and Tsujimoto Y (1999) Bcl-2 family proteins regulate the release of apoptogenic cytochrome $c$ by the mitochondrial channel VDAC. Nature 6735: 483-487

40. Narita M, Shimizu S, Ito T, Chittenden T, Lutz RJ, Matsuda $H$ and Tsujimoto $Y$ (1998) Bax interacts with the permeability transition pore to induce permeability transition and cytochrome $c$ release in isolated mitochondria. Proc. Natl. Acad. Sci. USA 25: 14681-14686

41. Nomura M, Shimizu S, Sugiyama T, Narita M, Ito T, Matsuda H and Tsujimoto $Y$ (2003) 14-3-3 interacts directly with and negatively regulates pro-apoptotic Bax. J. Biol. Chem. 3: 2058-2065

42. Linseman DA, Butts BD, Precht TA, Phelps RA, Le SS, Laessig TA, Bouchard RJ, Florez-McClure ML and Heidenreich KA (2004) Glycogen Synthase Kinase-3beta phosphorylates Bax and promotes its mitochondrial localization during neuronal apoptosis. J. Neurosci. 24: 9993-10002 